\title{
Comparison between in vitro embryo production using Y-sorted sperm and timed artificial insemination with non-sorted sperm to produce crossbred calves
}

\author{
Alexandre de Oliveira Bezerra ${ }^{a}$, Alessandra Corallo Nicacio ${ }^{\mathrm{b}}$, \\ Gilberto Romeiro de Oliveira Menezes ${ }^{\mathrm{b}}$, Rodrigo da Costa Gomes ${ }^{\mathrm{b}}$, \\ Luiz Otavio Campos da Silva ${ }^{\mathrm{b}}$, Nathália Alves de Souza Rocha-Frigoni ${ }^{\mathrm{c}}$, \\ Gisele Zoccal Mingoti ${ }^{c}$, Beatriz Caetano da Silva Leão ${ }^{c}$, \\ Eliane Vianna da Costa e Silva ${ }^{\mathrm{a}}$, Ériklis Nogueira ${ }^{\mathrm{a}, \mathrm{b}, \mathrm{d}, *}$ \\ a Programa de Pós-Graduação em Ciências Veterinárias, UFMS, Av. Senador Felinto Muller, 2443, 79070-900, Campo Grande, MS, Brazil \\ ${ }^{\mathrm{b}}$ Embrapa Gado de Corte, Av. Rádio Maia, 830, 79106-550, Vila Popular, Campo Grande, MS, Brazil \\ ' UNESP Araçatuba, FMV, Rua Clovis Pestana, 793, Araçatuba, SP, Brazil \\ ${ }^{\mathrm{d}}$ Embrapa Pantanal, Rua 21 de Setembro, 1880, 79320-900, Corumbá, MS, Brazil
}

\section{A R T I C L E I N F O}

\section{Keywords:}

Cryopreservation

Crossbreeding

FTET

FTAI

Nelore-Angus

\begin{abstract}
A B S T R A C T
Due to the increasing use of in vitro embryo production (IVEP) and the importance of crossbreeding for beef production, pregnancy rates of Nelore recipients were evaluated following Fixed Time Embryo Transfer with fresh or vitrified IVEP embryos produced with Y-sorted sperm of Angus bulls (B. taurus) or Fixed Time Artificial Insemination using non-sorted sperm. For IVEP in Experiment 1, oocytes were obtained using Ovum Pick Up (OPU) ( $n=84$ embryos) or from ovaries from a slaughterhouse (SLAUGHTER, $n=66$ embryos). In Experiment 2, with oocytes obtained by OPU, IVEP embryos were fresh (FRESH, $n=271$ ) or after vitrification/warming (VITRIFIED, $n=79)$ and PR was compared with FTAI $(n=239)$. In Experiment 1 , cleavage rates were $63.8 \%$ and $39.1 \%$ for OPU and SLAUGHTER groups, respectively $(P=0.02)$, and blastocyst rates were $30.5 \%$ and $14.7 \%$, respectively $(P=0.09)$. The PR was similar when considering the source of oocytes $(\mathrm{OPU}=35.7 \%$; SLAUGHTER $=25.8 \% ; P=0.17)$. In Experiment 2 , there was no difference in PR for FRESH or VITRIFIED embryos ( $34.3 \%$ and $30.4 \%$, respectively, $P=0.72$ ), but lesser than FTAI $(47.7, P=0.002)$. It is concluded that the IVEP with Y-sorted sperm associated with vitrification or embryos produced with oocytes from different sources did not affect PR when there was transfer of crossbred embryos into recipients, and can optimize large-scale application of IVEP technology; however, FTAI pregnancy rates with non-sex sorted sperm were greater.
\end{abstract}

\section{Introduction}

The increasing food demands have challenged the livestock sector to develop new technologies that enable greater meat production in less time and at a less cost (Blondin, 2015). In Brazil, Nelore is the most common breed of cattle, because it can easily adapt

\footnotetext{
*Corresponding author at: Embrapa Gado de Corte, Av. Rádio Maia, 830, 79106-550, Vila Popular, Campo Grande, MS, Brazil.

E-mail address: eriklis.nogueira@embrapa.br (É. Nogueira).
} 
to the environment, and some consensus that the use of well-designed crossbreeding can improve the production rates of herds (Morotti et al., 2014; Marinho et al., 2015; Rodrigues et al., 2015).

Fixed-time artificial insemination (FTAI) is a widely used reproductive biotechnological technique that favors production of genetically superior animals, and when used there is an average pregnancy rate of approximately 50\% (Sa Filho et al., 2009). For this reason, the optimization of FTAI and the breeding of genetically superior animals (for production characteristics) may allow for the use of FTAI in commercial systems and result in a more favorable cost/benefit ratio. Results of previous studies, however, indicate that the use of sex-sorted semen in FTAI may negatively affect pregnancy rates (Grant and Chamley, 2007) because of lesser number of sperm per dose $\left(2 \times 10^{6} /\right.$ dose $)$ or damage in sperm due to process of sexing, causing decreased fertility (Frijters et al., 2009).

For both beef and dairy cattle, the use of in vitro embryo production (IVEP) has the potential to maximize the genetic contribution of the most productive animals, improving reproductive efficiency by reducing the generation interval (Pontes et al., 2011). The large ovarian follicular population for the Nelore breed aids in maintaining Brazil's status as the greatest producer of in vitro-derived cattle embryos in the world (Pontes et al., 2009). The expansion of IVEP has been enhanced because of the favorable oocyte production of individuals of the Nelore breed because as the number of oocytes recovered increases, embryo production and resulting pregnancies increase (Pontes et al., 2011).

The impact of IVEP has expanded through the use of sex-sorted sperm of animals with high genetic merit, which has improved the cost/benefit ratio for this technique (Rodrigues, 2014). The process involved in sexing and freezing semen decreases sperm fertility in comparison with conventional semen (Wheeler et al., 2006; Xu et al., 2009; Seidel, 2014; Liu et al., 2015), so the most common use for sex-sorted sperm in Brazil is IVEP (Wheeler et al., 2006; Morotti et al., 2014).

Another major challenge for the embryo industry is cryopreservation, and frequently, Brazil has a shortage of recipient cows capable of receiving an embryo, primarily because of the large number of embryos produced (Pontes et al., 2011). This issue could result in discarding of embryos when cryopreservation is not used. For this reason, alternatives, including the cryopreservation of embryos and synchronization for fixed-time embryo transfer (FTET), are used to optimize and maximize the use of embryos produced in vitro, with similar pregnancy rates with fresh or cryopreserved IVEP embryos (Marinho et al., 2015). Additionally, among the factors considered in FTET programs, the recipients are obviously the major determining factor for the successful use of this biotechnology (Spell et al., 2001).

As an alternative to increase production of crossbred beef calves, there were two objectives in the present study: 1) Comparison of the production and PR of embryos with sex-sorted sperm from oocytes obtained from the aspiration of follicles of live animals (OPU) or after slaughter; 2) Comparison of PR of embryos produced with sex-sorted sperm and transferred fresh or after vitrification/ warming with artificial insemination using non-sorted sperm in Nelore recipients.

\section{Materials and methods}

All animal procedures were approved by the Animal Care and Use Committee of the EMBRAPA (Protocol 02-2015), Brazil.

\subsection{Experimental design}

\subsubsection{Experiment 1}

Cumulus oocyte complexes (COCs) were obtained using follicular aspiration procedures from ovaries obtained at a slaughterhouse (SLAUGHTER Group) or using follicular aspiration guided ultrasonography procedures (OPU group) from nine Nelore heifers, and by using IVEP with sex sorted sperm from an Angus bull.

\subsubsection{Experiment 2}

There were three groups for Experiment 2: FRESH, VITRIFIED in vitro embryos (IVEP with oocytes obtained using OPU from 23 Nelore donors and sex-sorted sperm from two Angus bulls), and FTAI ( $n=239)$, with cows inseminated at fixed time, with non sexsorted sperm from the same Angus bulls used in IVEP.

\subsection{In vitro Embryo production}

All chemicals used in this study were purchased from Sigma-Aldrich (St. Louis, MO, USA) unless otherwise stated.

\subsubsection{Cumulus-oocyte complexes (COC) recovery: slaughterhouse tissue}

In the SLAUGHTER group (Experiment 1), Nelore cow ovaries of unknown origin were collected shortly after slaughter and transported to the laboratory in a thermostatic container containing phosphate-buffered saline (Nutricell, Campinas, São Paulo, Brazil) at a temperature between 35 to $37^{\circ} \mathrm{C}$. Two collections were conducted on different days. Follicles between 3 and 8 mm in diameter were aspirated with an $18 \mathrm{G}$ needle connected to a $20 \mathrm{ml}$ syringe. The COCs were placed in $50 \mathrm{ml}$ conical tubes in a water bath at $30{ }^{\circ} \mathrm{C}$ for decanting at the time of oocyte selection, with total time from the slaughter of the animals to the end of the aspirations of approximately $6 \mathrm{~h}$.

\subsubsection{Cumulus-oocyte complexes (COC) recovery: ovum pick-up}

Cumulus-oocyte complexes (COCs) were obtained using ultrasonic-guided follicular aspiration procedures for ovum pick-up (OPU) from nine estrous-cyclic Nelore heifers, in Experiment 1, aged 18 to 36 months with body condition scores (BCS) between 2.5 
and 3 (on a scale of 1 to 5), and from 23 cows in Experiment 2 aged 24 to 80 months with BCS between 2.5 and 4 , from two herds which had sustained programs for genetic selection and evaluation. The animals were kept in Brachiaria brizantha pastures with ad libitum mineral supplementation. Two follicular aspirations were performed with an interval of 22 days between them and regardless of the donor's estrous cycle stage.

Before each collection, any feces present were removed from the rectum, and the perineal region was subsequently cleaned with paper towels. Epidural anesthesia was subsequently performed using 2\% lidocaine (Anesthetic L, Eurofarma, Campo Belo, São Paulo, Brazil) at a dose of $0.2 \mathrm{mg} / \mathrm{kg}$ to reduce peristaltic movements and donor cow discomfort. Oocyte collection was performed using ultrasonic equipment (SSD-500, Aloka Co., Tokyo, Japan) with a convex7.5 MHz transducer coupled to a special suction guide (WTA, Cravinhos, São Paulo, Brazil). Follicles with diameters between 2 and $8 \mathrm{~mm}$ were punctured with a $20 \mathrm{G}$ disposable needle (Terumo, São Paulo, Brazil) connected by a silicone tube $(1.20 \mathrm{~m}$ ) to a $50-\mathrm{mL}$ conical tube and to the vacuum system used for aspiration (WTA, Cravinhos, São Paulo, Brazil), which functioned with a negative pressure of $70 \mathrm{~mm} \mathrm{Hg}$.

\subsubsection{Oocyte selection and in vitro maturation (IVM)}

After obtaining the oocytes, the material collected was filtered using an Emcon ${ }^{\varpi}$ filter with phosphate-buffered saline (PBS) solution with $10 \%$ fetal calf serum (FCS). The immature COCs recovered were morphologically classified as viable or non-viable. The COCs were considered viable if they had multiple compact layers of cumulus cells and a homogeneous ooplasm and non-viable if they appeared bare or pyknotic with a heterogeneous ooplasm and apoptotic vesicles.

The viable COCs were washed three times in the flushing medium and once in an in vitro maturation medium (IVM) [TCM-199 supplemented with $10 \%$ (v:v) (FCS), $0.2 \mathrm{mMof}$ sodium pyruvate, $25 \mathrm{mM}$ of sodium bicarbonate, $50 \mathrm{mg} / \mathrm{mL} \mathrm{of} \mathrm{amikacin,} 0.5 \mathrm{mg} / \mathrm{mL}$ of follicle-stimulating hormone (FSH, Folltropin-V; Bioniche Animal Health, Ontario, Canada) and $100 \mathrm{UI} / \mathrm{mL}$ of human chorionic gonadotropin (hCG, Vetecor; Hertape Calier). The selected COCs were subsequently transferred to wells containing $400 \mu \mathrm{L}$ of the maturation medium under mineral oil (at a concentration of $30-40$ oocytes per well) and then incubated for $22-24$ hours at $38.5{ }^{\circ} \mathrm{C}$, with $5 \% \mathrm{CO}_{2}$ and saturated humidity.

\subsubsection{Semen preparation and in vitro fertilization (IVF)}

The fertilization medium consisted of Tyrode's albumin lactate pyruvate (TALP), as described by (Parrish et al., 1988), containing $0.2 \mathrm{mM}$ sodium pyruvate, $6 \mathrm{mg} / \mathrm{mL}$ bovine serum albumin (BSA), $25 \mathrm{mM}$ sodium bicarbonate, $13 \mathrm{mMof}$ sodium lactate, $50 \mathrm{mg} / \mathrm{mL}$ amikacin, $40 \mu \mathrm{L} / \mathrm{mL}$ penicillamine-hypotaurine-epinephrine (PHE) solution (with final concentrations of $20 \mu \mathrm{M}$ of penicillamine, 10 $\mu \mathrm{M}$ hypotaurine and $2 \mu \mathrm{M}$ epinephrine) and $10 \mathrm{mg} / \mathrm{mL}$ heparin. Straws containing cryopreserved Y-sorted sperm from two Aberdeen Angus bulls known to be fertile were thawed in a $37{ }^{\circ} \mathrm{C}$ water bath for $30 \mathrm{~s}$. Centrifugation in a Percoll discontinuous gradient (200 $\mu \mathrm{L}$ of $45 \%$ over $200 \mu \mathrm{L}$ of $90 \%$ ) was performed at $1000 \times g$ for $7 \mathrm{~min}$ for the selection of motile sperm and removal of diluent and seminal plasma. The supernatant was discarded, and the pellet containing viable sperm was re-suspended in $1 \mathrm{~mL}$ of fertilization medium and centrifuged again at $350 \times g$ for $5 \mathrm{~min}$. The recovered sperm were evaluated for motility and concentration using light microscopy. The sperm were then diluted in fertilization medium to achieve a final concentration of $1 \times 10^{6} \mathrm{sperm} / \mathrm{mL}$.

The mature COCs were washed once in TALP sperm medium and once in fertilization medium. The COCs were then transferred to a drop of fertilization medium (at a concentration of 15-20 COCs per drop in $90 \mu \mathrm{L}$ of fertilization medium) and co-incubated with sperm for 18-22 h using the same conditions used for IVM. The day of fertilization was considered Day 0 (D0).

\subsubsection{In vitro Culture (IVC)}

The medium used for in vitro cultivation (IVC) consisted of modified medium synthetic oviductal fluid (SOF), as described by Vajta et al. (1999), supplemented with $50 \mathrm{mg} / \mathrm{mL}$ of amikacin, $5 \mathrm{mg} / \mathrm{mL}$ of BSA and $2.5 \%$ of fetal bovine serum (FBS) (v:v). After fertilization (D0), the cumulus cells were removed by successive pipetting. The probable zygotes were washed once in TALP sperm medium, once in 50\% TALP sperm medium plus 50\% modified SOF medium and once again modified SOF medium. Subsequently, the probable zygotes were transferred (in groups of 25) to $100 \mu \mathrm{L}$ droplets of modified SOF medium. The embryos were cultured at $38.5^{\circ} \mathrm{C}, 5 \% \mathrm{CO}_{2}$ and saturated humidity for 7 days. The culture medium was partially replaced with $50 \mu \mathrm{L}$ of fresh modified SOF medium at $48 \mathrm{~h}$ post fertilization (D3) when the rate of cleavage was assessed.

\subsubsection{Evaluation of embryos}

The rates of embryonic development to the blastocyst stage were evaluated on day 7 (D7) of the culture. The embryos were classified according to the International Embryo Technology Society (IETS) criteria (Bo and Mapletoft, 2013). Only embryos classified as quality grade I were transferred fresh or post-vitrification/warming to the recipients.

\subsection{Vitrification and warming}

All materials used in the vitrification/warming procedure were purchased from Ingámed Ltda. (Perobal, PR, Brazil), including the vitrification solutions (VI-I and VI-II), incubation solutions (DV-I, DV-II and DV-III) and vitrification strips (Vitri-Ingá) and the plastic sheaths. A two-step technique was used for the vitrification of the blastocysts according to (Almodin et al., 2010; Rocha-Frigoni et al., 2014). Vitrified embryos were thawed by immersing each straw in a water bath at $37^{\circ} \mathrm{C}$ for $10 \mathrm{~s}$. After warming, the embryos were cultured for $2 \mathrm{~h}$ in SOF medium supplemented with $2.5 \%$ FCS and $5 \mathrm{mg} / \mathrm{mL}$ of BSA in an atmosphere with $5 \% \mathrm{CO}_{2}$ and saturated humidity. Embryo survival after warming was evaluated by the rate of re-expansion after $2 \mathrm{~h}$ of culture. Only viable re-expanded embryos were loaded individually in $0.25-\mathrm{ml}$ French straws for subsequent transfer to recipients. 


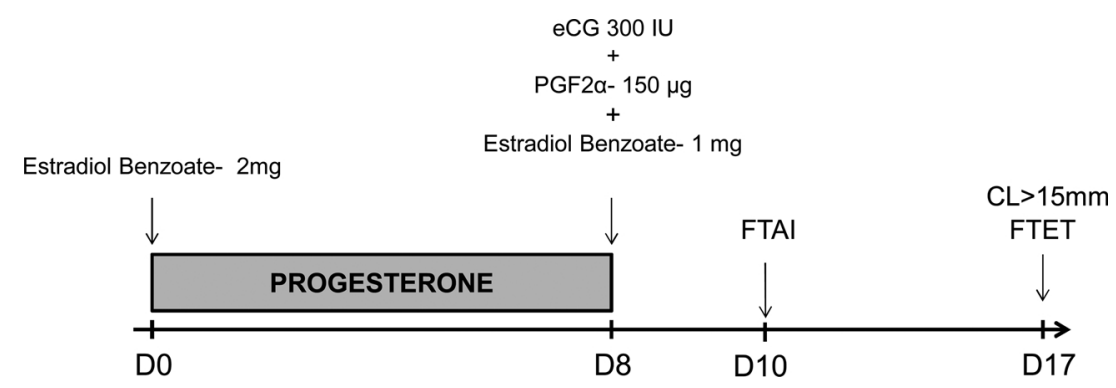

Fig. 1. Estrous synchronization protocol for FTET and FTAI used in Experiments 1 and 2; On the first day of the protocol (D0), an intravaginal P4 device (Cronipress ${ }^{\circledast}$ Mono Dose M-24, Biogenesis Bagó, Curitiba, Paraná, Brazil) was administered along with $2 \mathrm{mg}$ of estradiol benzoate (Estrogin, Biofarm, Jaboticabal, São Paulo, Brazil), D8- withdrawal of the P4 device, concomitant with the application of PGF2 $\alpha$ (150 $\mu$ d d-cloprostenol, i.m. Prolise $^{\circledR}$, Arsa, Buenos Aires, Argentina), $1 \mathrm{mg}$ of estradiol benzoate (Estrogin, Biofarm, Jaboticabal, São Paulo, Brazil) and 300 IU of eCG (Folligon ${ }^{\circledR}$ 5000IU, MSD, São Paulo, Brazil). D10 was considered the day of estrus; FTAI was performed on D10, and FTET was performed on D17.

\subsection{Fixed-time embryo transfer (FTET) and fixed-time artificial insemination (FTAI)}

Multiparous Nelore cows $(n=589$ ) with BCS ranging from 2.5 to 3.5 ( 1 to 5 ) at an average of 45 days post-partum, without uterine disease and with stage of estrous cycle unknown were used as recipients (Experiments 1 and 2) or for FTAI (Experiment 2). The estrous synchronization protocol for FTET and FTAI was performed as follows: on the first day of the protocol (D0), an intravaginal P4 device (Cronipress ${ }^{\circledast}$ Mono Dose M-24, Biogenesis Bagó, Curitiba, Paraná, Brazil) was administered along with 2 mg of estradiol benzoate (Estrogin, Biofarm, São Paulo, Brazil), D8- withdrawal of the P4 device, concomitant with the administration of $\mathrm{PGF}_{2 \alpha}\left(150 \mu \mathrm{g}\right.$ d-cloprostenol, i.m.Prolise ${ }^{\circledR}$, Arsa, Buenos Aires, Argentina), $1 \mathrm{mg}$ of estradiol benzoate (Estrogin, Biofarm, São Paulo, Brazil) and 300 IU of eCG (Folligon 5000 IU, MSD, São Paulo, Brazil). Day of estrus was considered to be D10; For FTAI, the females were subjected to the same protocol used for FTET, but the AI was performed on D10, 48 to $50 \mathrm{~h}$ after implant removal, with conventional cryopreserved semen (non sex sorted) from the same bulls used for the IVEP (Fig. 1).

For FTET, ET was performed on animals if there was a corpus luteum (CL) diameter of at least 15 mm on D17, which was evaluated by ultrasonography. Pregnancy was detected using ultrasonic equipment (Mindray DP-20VET, Shenzhen, Chin) with a linear 7.5 MHz transducer, 60 days after the ET or FTAI.

\subsection{Statistical analysis}

The statistical analysis was performed by using a generalized linear mixed model using the SAS GLIMMIX procedure with binomial distribution (SAS Institute, Inc., Cary, NC, USA). The variables included in the model used to evaluate the pregnancy rate were as follows: treatment (OPU or SLAUGHTER in Experiment1, and FRESH, VITRIFIED or FTAI, in Experiment 2), bull, BCS of the cows, technician and interactions. The variables in which the effect was not significant $(P<0.05)$ were removed from the model.

The probability of pregnancy in relation to the CL diameter in the FTET group in Experiment 1 was assessed using logistic regression using the SAS LOGISTIC procedure (SAS Institute, Inc., Cary, NC, USA). Differences with $P<0.05$ were considered significant, whereas trends were only considered significant when $0.1>P \geq 0.05$. The data are presented as the mean \pm standard error (SE).

\section{Results}

\subsection{Experiment 1}

The total number of viable oocytes obtained through OPU was 279 (mean of 15.5/ donor), while aspiration of ovaries obtained from slaughterhouse (SLAUGHTER) resulted in collection of 448 oocytes (mean of 17.9 oocytes per pair of ovaries). There was a trend for a greater percentage development to the blastocyst stage in the OPU group (30.5\%) compared to the SLAUGHTER group (14.7\%, $P=0.09$ ). From the total number of embryos developed to the blastocyst stage, 66 were transferred in the SLAUGHTER group and 84 in the OPU group (with one embryo not being transferred).

The pregnancy rate did not differ for group (OPU - 35.7\%, SLAUGHTER - 25.8\%, $P=0.17$, Table 1). The recipients had a CL diameter of $21.5 \pm 3.45 \mathrm{~mm}$, with a proportion of compact and cavitary corpora lutea of $61.3 \%$ and $38.7 \%$, respectively. There was no effect of the size of the CL on the probability of pregnancy $(P=0.44)$. Furthermore, the type of CL did not affect the pregnancy rate (compact $=25.0 \%$; cavitary $=29.3 ; P=0.82$ ).

\subsection{Experiment 2}

The total number of viable oocytes obtained using OPU was 1064 (with a mean of 23.13 oocytes/donor), with a variation between individuals ranging from 0 to 82 oocytes per OPU session. The percentages for cleavage and blastocyst formation were of $52.0 \%$ and 
Table 1

Cleavage, blastocyst and pregnancy rates with differing oocytes sources in Experiment 1.

\begin{tabular}{lllll}
\hline & OPU & SLAUGHTER & Standard Error (SE) & $P$ \\
\hline Cleavage (\%) & $63.80(178 / 279)$ & $39.06(175 / 448)$ & 10.22 & 0.0209 \\
Blastocyst (\%) & $30.47(85 / 279)$ & $14.73(66 / 448)$ & 9.13 & 0.0892 \\
Pregnancy (\%) & $35.71(30 / 84)$ & $25.76(17 / 66)$ & 0.39 & 0.170 \\
\hline
\end{tabular}

SLAUGHTER- Cumulus-oocyte complexes (COCs) were obtained from Slaughterhouse ovaries.

OPU- Cumulus-oocyte complexes (COCs) were obtained by ultrasound-guided follicular aspiration for ovum pick-up (OPU).

$(P<0.05)$.

35.7\%, respectively, with 271 embryos developing and being transferred in FRESH group and 109 embryos submitted to vitrification. The re-expansion rate after warming was 72.5\% (79/109), with 79 grade I vitrified/warmed embryos transferred in VITRIFIED group.

There was no difference in the pregnancy rate for embryos transferred fresh $(35.42 \%)$ or vitrified $(30.38 \% ; P=0.79$, Table 2$)$, however, there was a difference from the FTAI group $(47.7 \%, P=0.002)$. The pregnancy rate per bull did not differ $(P>0.05)$ for either FTAI or FTET.

\section{Discussion}

Females of the Nelore breed are characterized by the relatively large oocyte production as compared with most other breeds: producing an average of 25 to 30 oocytes per OPU session (Pontes et al., 2009, 2011), which is greater than the taurine cattle breeds (Pontes et al., 2009). In the present study, the mean number of viable oocytes per OPU procedure (15. 5 in Experiment 1) was less than that previously reported. This result may be a consequence of the donor animals being heifers. Notably, there has been considerable individual variation in the number of follicles and oocytes of Nelore females (Pontes et al., 2011). This finding was verified from results in the present study with the number of oocytes per OPU session ranging from 0 to 82 in Experiment 2.

The rates for in vitro embryonic development after the fertilization of oocytes obtained by OPU were similar to those observed by other researchers where there was use of both conventional semen (blastocyst rate: 32.7\%; (Varago et al., 2006)) and sex-sorted sperm (blastocyst rate: 33.2\% (Xu et al., 2006)). From other studies, there are reports that there is a lesser percentage of development to the blastocyst stage when there is in vitro fertilization of oocytes derived from ovaries of slaughtered animals and fertilized with $\mathrm{Y}$ sex-sorted sperm (12.2\%) when compared with conventional semen (20.1\%) (Wilson et al., 2006). The lesser cleavage and percentage development to the blastocyst stage with use of slaughterhouse ovarian oocytes may have resulted from the time of about $6 \mathrm{~h}$ from ovary collections, transportation of ovaries, and aspiration in laboratory which has been previously reported to reduce the quality of oocytes (Satrapa et al., 2011).

It is more difficult to maintain sanitary rigor (Ponsart and Pozzi, 2013) and control of the genetic merit of animals, with the use of ovaries from the slaughterhouse, but the use of this approach can contribute to the reduction of the costs of cattle IVEP by eliminating the need for in vivo Ovum Pick-up (Wilson et al., 2006) mainly for the production of crossbred animals with oocytes from Bos indicus donors and semen from genetically superior Bos taurus bulls. This approach allows for intensification of meat production in different systems, because crossbred calves weigh $10 \%$ to $15 \%$ more at weaning (Rodrigues et al., 2015).

Zhang et al. (2003) reported that there was no a significant difference in the percentage of development to the blastocyst stage with the use of sex-sorted (20.3\%) and non sex-sorted (22.3\%) semen. Malard et al. (2015), reported that there were lesser percentages of development to the blastocyst stage when comparing inseminations with sex-sorted semen and non sex-sorted semen (29.0\% compared with $37.1 \%$, respectively); however, there was a greater pregnancy rate for IVEP embryos where there was the use of sex-sorted sperm as compared with non sex-sorted sperm (36.9\% compared with 30.1\%). This inconsistency in results may be explained by the lesser fertilization capacity of the sperm after the sexing process (Grant and Chamley, 2007). The previously reported results were sometimes inconsistent or conflicting, and differences among studies regarding sperm sexing processes, oocyte maturation and fertilization and embryo cultivation made interpretation of the results difficult (Mikkola et al., 2015).

In addition to the differences in the procedures used by the different research groups, several other factors may also affect oocyte viability for IVEP. The effect of the bull used on the rate of production of blastocysts should be considered, as the efficiency of IVEP can vary depending on bull factors (Xu et al., 2006; Bayeux et al., 2015). This variation seems to be greater with the use of sex-sorted

Table 2

Pregnancy rate for in vitro produced embryos using Y-sorted sperm or FTAI with non-sex sorted sperm in Experiment 2.

\begin{tabular}{lllll}
\hline & FTET-FRESH & FTET-VITRIFIED & FTAI & $P$ \\
\hline $\mathrm{n}$ & 271 & 79 & 239 & $47.70(114 / 239)^{\mathrm{a}}$ \\
Pregnancy rate & $35.42(96 / 271)^{\mathrm{b}}$ & $30.38(24 / 79)^{\mathrm{b}}$ & 0.0019 \\
\hline
\end{tabular}

FTET-FRESH- in vitro embryos produced with sex-sorted semen and transferred fresh at fixed time.

FTET-VITRIFIED- in vitro embryos produced with sex-sorted semen and transferred after vitrification and warming at fixed time.

FTAI- Fixed time artificial insemination with non-sorted semen.

Different superscript letter $\mathrm{a}, \mathrm{b}$ in the same line indicate significant difference $(\mathrm{P}<0.05)$. 
semen, affecting the percentage of development to the blastocyst stage (Palma et al., 2008). In the present study, however, there were no differences in results due to bull factors.

In the present study, the pregnancy rates when FTET was performed with fresh embryos or vitrified embryos were similar $(P=$ 0.79, Table 2). Results of several studies indicate that there are negative effects of cryopreservation on embryo development after warming (Cavusoglu et al., 2016), but the effects of cryopreservation may vary with animal species, breed, origin (in vitro or in vivo), lipid composition of the embryo, size and stage of development, quality of the embryo/oocyte, cell permeability, toxicity and osmotic properties of cryoprotectants and cooling and warming rates (Almiñana and Cuello, 2015). In contrast, results of other studies indicate that there can be acceptable pregnancy rates after FTET of embryos derived with use of IVEP and fertilization with sex-sorted semen, both fresh and vitrified (Xu et al., 2006; Pontes et al., 2011; Trigal et al., 2012) or only vitrified/warmed (Marinho et al., 2015). There has been significant recent progress in enhancing embryo culture and cryopreservation procedures, regardless of the use of sex-sorted semen (Xu et al., 2009). This progress has allowed the expansion of the use of this technique on a large scale, optimizing the use of recipients and allowing access to recipients that are in distant locations, as substantiated by the results of the present study.

Even though there were lesser pregnancy rates in the FTET group with the use of sex-sorted semen compared to the FTAI group with use of conventional semen, the proportion of animals born with the desired sex may be advantageous for use of this technique. The next phase of the present line of research will be evaluation of the economic efficiency with use of these techniques when there is production of the animals being used for slaughter.

Multiparous Nelore cows with acceptable pregnancy rates were used as recipients in the present study. The selection of recipients was judicious, and the use of animals with larger CL diameters may have contributed to the desirable pregnancy rates (Binelli et al., 2001; Nogueira et al., 2012; Gonella-Diaza et al., 2013), as the serum concentration of progesterone (P4) is a reflection of the development and size of the luteal mass (Spell et al., 2001), and consequently in the survival of the concept and maintenance of pregnancy (Binelli et al., 2001). Factors such as blood flow, the presence of luteotropic or luteolytic agents, growth factors and the availability of precursors for P4 biosynthesis (Skarzynski et al., 2008), and lactation may interfere with P4 production. In the present study, there was no association between the CL diameter and pregnancy rate, most likely due to selection of recipients with corpora lutea greater than $15 \mathrm{~mm}$ in diameter. This factor supports the importance of assessing CL diameter at the time of transfer, as indicated based on results in various studies (Spell et al., 2001; Looney et al., 2006; Gonella-Diaza et al., 2013).

Additionally, there was no effect of CL type (cavitary or compact) on the pregnancy rate in the present study, and according to (Nogueira et al., 2012; Barreiros et al., 2006), the morphological differences between different types of corpora lutea do not affect the production of $\mathrm{P} 4$ and, consequently, do not affect the pregnancy rate. In the present study, there was a similar result when there were cavitary and compact corpora lutea with no effect on pregnancy rate being observed.

\section{Conclusion}

Results of the present study indicate that multiparous Nelore cows used as recipients produce acceptable pregnancy rates, with use of FTET, without effects of corpora lutea size on pregnancy rates. The conducting of IVEP with use of sex-sorted semen, associated with vitrification when there were different ovarian sources for oocytes used, resulted in acceptable pregnancy rates. The production of the crossbred embryos using these techniques could allow for optimization of large-scale application of the IVEP technology even though FTAI pregnancy rates with use of non-sex sorted semen were greater than when there was FTET with sex-sorted semen to produce embryos.

\section{Funding information}

This research was supported by EMBRAPA (Grant \# 02.13.14.011.00.00; Cow-cal innovation aiming precocious steers production, Brazil), and Coordination for the Improvement of Higher Education Personnel (CAPES), Post-Graduation Program of Veterinary Sciences at University Federal of Mato Grosso do Sul (UFMS), Brazil.

\section{Acknowledgements}

Betagen Laboratories for IVE production, and the farmers for their help and support for this study.

\section{References}

Almiñana, C., Cuello, C., 2015. What is new in the cryopreservation of embryos. Anim. Reprod. 12, 418-427.

Almodin, C.G., Minguetti-Camara, V.C., Paixao, C.L., Pereira, P.C., 2010. Embryo development and gestation using fresh and vitrified oocytes. Hum. Reprod. 25, 1192-1198.

Barreiros, T.R.R., Blaschi, W., Borsato, E.A., Ludwig, H.E., Meira Da Silva, D.R., Seneda, M.M., 2006. Comparasion the pregnancy rates between bovine recipients with cavitary or compact corpus luteum after cloprostenol or fixed time embryo transfer. Semina: Ciências Agrárias 27, $657-664$.

Bayeux, B., Monteiro, F., Batista, E., Vieira, L., Accorsi, M., Campanholi, S., Dias, E., Souza, A., Baruselli, P., 2015. In vitro embryo production of Nelore (Bos indicus) donors submitted to a commercial program during 12 months. Anim. Reprod. 12 715-715.

Binelli, M., Thatcher, W.W., Mattos, R., Baruselli, P.S., 2001. Antiluteolytic strategies to improve fertility in cattle. Theriogenology 56, $1451-1463$.

Blondin, P., 2015. Status of embryo production in the world. Anim. Reprod. 12, 356-358.

Bo, G., Mapletoft, R., 2013. Evaluation and classification of bovine embryos. Anim. Reprod. 10, 344-348.

Cavusoglu, T., Popken, J., Guengoer, T., Yilmaz, O., Uyanikgil, Y., Ates, U., Baka, M., Oztas, E., Zakhartchenko, V., 2016. Ultra-structural alterations in in vitro produced four-cell bovine embryos following controlled slow freezing or vitrification. Anat. Histol. Embryo. 45, $291-307$. 
Frijters, A.C., Mullaart, E., Roelofs, R.M., van Hoorne, R.P., Moreno, J.F., Moreno, O., Merton, J.S., 2009. What affects fertility of sexed bull semen more, low sperm dosage or the sorting process? Theriogenology 71, 64-67.

Gonella-Diaza, A., Holguín, G., Montaña, D., Valbuena, D., 2013. Corpus luteum diameter and embryo developmental stage are associated with pregnancy rate: data analysis from 17, 521 embryo transfer s from a commercial in vitro bovine embryo production program. Anim. Reprod. 10, 106-111.

Grant, V.J., Chamley, L.W., 2007. Sex-sorted sperm and fertility: an alternative view. Biol. Reprod. 76, 184-188.

Liu, X., Hu, T., Sun, W., Hao, H., Liu, Y., Zhao, X., Zhu, H., Du, W., 2015. Comparison of the developmental competence and quality of bovine embryos obtained by in vitro fertilization with sex-sorted and unsorted semen from seven bulls. Livest. Sci. 181, 263-270.

Looney, C.R., Nelson, J.S., Schneider, H.J., Forrest, D.W., 2006. Improving fertility in beef cow recipients. Theriogenology 65, $201-209$.

Malard, P., Oliveira, L., Santos, A., Queiroz, L., 2015. Comparison of IVF embryo production and pregnancy, using conventional sperm and sex-sorted sperm in cattle. Anim. Reprod. 12 681-681.

Marinho, L.S., Sanches, B.V., Rosa, C.O., Tannura, J.H., Rigo, A.G., Basso, A.C., Pontes, J.H., Seneda, M.M., 2015. Pregnancy rates to fixed embryo transfer of vitrified IVP bos indicus, bos taurus or bos indicus x bos taurus embryos. Reprod. Dom. Anim. 50, 807-811.

Mikkola, M., Andersson, M., Taponen, J., 2015. Transfer of cattle embryos produced with sex-sorted semen results in impaired pregnancy rate and increased male calf mortality. Theriogenology 84, 1118-1122.

Morotti, F., Sanches, B.V., Pontes, J.H., Basso, A.C., Siqueira, E.R., Lisboa, L.A., Seneda, M.M., 2014. Pregnancy rate and birth rate of calves from a large-scale IVF program using reverse-sorted semen in bos indicus, bos indicus-taurus, and bos taurus cattle. Theriogenology 81, 696-701.

Nogueira, E., Cardoso, G.S., Marques Junior, H.R., Dias, A.M., Ítavo, L.C.V., Borges, J.C., 2012. Effect of breed and corpus luteum on pregnancy rate of bovine embryo recipients. Rev. Bras. Zootec. 41, 2129-2133.

Palma, G., Olivier, N., Neumüller, C., Sinowatz, F., 2008. Effects of sex-sorted spermatozoa on the efficiency of in vitro fertilization and ultrastructure of in vitro produced bovine blastocysts. Anat. Histol. Embryo. 37, 67-73.

Parrish, J., Susko-Parrish, J., Winer, M., First, N., 1988. Capacitation of bovine sperm by heparin. Biol. Reprod. 38, 1171-1180.

Ponsart, C., Pozzi, N., 2013. Sanitary requirements for bovine gametes and embryos in international trade. Anim. Reprod. 10, $283-296$.

Pontes, J.H., Nonato-Junior, I., Sanches, B.V., Ereno-Junior, J.C., Uvo, S., Barreiros, T.R., Oliveira, J.A., Hasler, J.F., Seneda, M.M., 2009. Comparison of embryo yield and pregnancy rate between in vivo and in vitro methods in the same nelore (bos indicus) donor cows. Theriogenology 71, 690-697.

Pontes, J.H., Melo Sterza, F.A., Basso, A.C., Ferreira, C.R., Sanches, B.V., Rubin, K.C., Seneda, M.M., 2011. Ovum pick up, in vitro embryo production, and pregnancy rates from a large-scale commercial program using nelore cattle (bos indicus) donors. Theriogenology 75, 1640-1646.

Rocha-Frigoni, N.A., Leao, B.C., Nogueira, E., Accorsi, M.F., Mingoti, G.Z., 2014. Reduced levels of intracellular reactive oxygen species and apoptotic status are not correlated with increases in cryotolerance of bovine embryos produced in vitro in the presence of antioxidants. Reprod. Fertil. Dev. 26, 797-805.

Rodrigues, C., 2014. Historical context of cattle embryo transfer technique in Brazil. Anim. Reprod. 11, 137-140.

Rodrigues, W.B., Wechsler, F.S., Nogueira, E., 2015. Reproductive performance and reconception of nellore cows according to their pure- or cross-bred calves. Reprod. Dom. Anim. 50, 431-436.

Sa Filho, O.G., Meneghetti, M., Peres, R.F., Lamb, G.C., Vasconcelos, J.L., 2009. Fixed-time artificial insemination with estradiol and progesterone for bos indicus cows II: strategies and factors affecting fertility. Theriogenology 72, 210-218.

Satrapa, R.A., Nabhan, T., Silva, C.F., Simoes, R.A., Razza, E.M., Puelker, R.Z., Trinca, L.A., Barros, C.M., 2011. Influence of sire breed (bos indicus versus bos taurus) and interval from slaughter to oocyte aspiration on heat stress tolerance of in vitro-produced bovine embryos. Theriogenology $76,1162-1167$.

Seidel, G., 2014. Update on sexed semen technology in cattle. Animal 8 (Suppl 1), 160-164.

Skarzynski, D.J., Ferreira-Dias, G., Okuda, K., 2008. Regulation of luteal function and corpus luteum regression in cows: hormonal control, immune mechanisms and intercellular communication. Reprod. Dom. Anim. 43 (Suppl 2), 57-65.

Spell, A.R., Beal, W.E., Corah, L.R., Lamb, G.C., 2001. Evaluating recipient and embryo factors that affect pregnancy rates of embryo transfer in beef cattle. Theriogenology 56, 287-297.

Trigal, B., Gomez, E., Caamano, J.N., Munoz, M., Moreno, J., Carrocera, S., Martin, D., Diez, C., 2012. In vitro and in vivo quality of bovine embryos in vitro produced with sex-sorted sperm. Theriogenology 78, 1465-1475.

Varago, F., Saliba, W., Alvim, M., Vasconcelos, A., Oliveira, C., Stahlberg, R., Lagares, M., 2006. Vitrification of in vitro produced zebu embryos. Anim. Reprod. Sci. 3, 353-358.

Vajta, G., Rindom, N., Peura, T.T., Holm, P., Greve, T., Callesen, H., 1999. The effect of media, serum and temperature on in vitro survival of bovine blastocysts after Open Pulled Straw (OPS) vitrification. Theriogenology 52, 939-948.

Wheeler, M.B., Rutledge, J.J., Fischer-Brown, A., VanEtten, T., Malusky, S., Beebe, D.J., 2006. Application of sexed semen technology to in vitro embryo production in cattle. Theriogenology 65, 219-227.

Wilson, R.D., Fricke, P.M., Leibfried-Rutledge, M.L., Rutledge, J.J., Penfield, C.M., Weigel, K.A., 2006. In vitro production of bovine embryos using sex-sorted sperm. Theriogenology 65, 1007-1015.

Xu, J., Guo, Z., Su, L., Nedambale, T.L., Zhang, J., Schenk, J., Moreno, J.F., Dinnyes, A., Ji, W., Tian, X.C., Yang, X., Du, F., 2006. Developmental potential of vitrified holstein cattle embryos fertilized in vitro with sex-sorted sperm. J. Dairy Sci. 89, 2510-2518.

Xu, J., Chaubal, S.A., Du, F., 2009. Optimizing IVF with sexed sperm in cattle. Theriogenology 71, 39-47.

Zhang, M., Lu, K.H., Seidel, G.E., 2003. Development of bovine embryos after in vitro fertilization of oocytes with flow cytometrically sorted, stained and unsorted sperm from different bulls. Theriogenology 60, 1657-1663. 